\title{
Hybrid Navigation Interface A Comparative Study
}

\author{
Philipp Stefan ${ }^{1}$, Joerg Traub ${ }^{1}$, Sandro M. Heining ${ }^{2}$, Christian Riquarts ${ }^{2}$, \\ Tobias Sielhorst ${ }^{1}$, Ekkehard Euler ${ }^{2}$, Nassir Navab ${ }^{1}$ \\ ${ }^{1}$ Chair for Computer Aided Medical Procedures (CAMP), I-16, Technische \\ Universität München, Boltzmannstraße 3, 85748 Garching b. München, Germany \\ ${ }^{2}$ Trauma Surgery Department, Klinikum Innenstadt, Ludwig Maximilian Universität \\ München, Nußbaumstraße 20, 80336 München, Germany \\ Email: stefanp@cs.tum.edu
}

\begin{abstract}
Since the introduction of computer aided surgery, many visualisation techniques for intraoperative navigation have been proposed. Systems employing multi planar reconstruction for the visualisation of volumetric imaging data are commercially available and frequently used. In these systems, three-dimensional biomedical data is generally displayed on a two-dimensional computer monitor as orthogonal planar sections defined by the orientation of a surgical instrument. In-situ visualisation, that was introduced as an alternative approach for intraoperative navigation, superimposes three-dimensional imaging data directly on the surgical object, typically using a stereoscopic display device, e.g. a head mounted display. In this paper, we compare monitor based navigation with video see-through augmented reality visualisation regarding performance and usability. Furthermore, we compare each with a hybrid of both systems that was recently introduced. We created an experimental setup to simulate an exemplary application for trauma and orthopedic surgery and conducted the experiment with three trauma surgeons with different levels of experience using all three approaches.
\end{abstract}

\section{Introduction}

The aims of computer aided surgery are the improvement of patient care by utilising computational tools during treatment. This includes the guidance of the surgeon during the intervention using intraoperative navigation systems, that visualise volumetric biomedical imaging data with respect to the orientation of surgical instruments.

Multi planar reconstruction (MPR) is a commonly used visualisation technique for such volumetric datasets. A tracked instrument defines planar sections through the reconstructed volume, that are visualised on a computer monitor display. Commercially available navigation systems, that employ MPR exist for more than a decade. The drawbacks of state-of-the-art navigation systems, based on preoperative or intraoperative imaging data, are, that a) the visualisation is focused on a small region of interest and does not give an overview of the surgical 
workspace, b) the guidance information based on three-dimensional data is presented on two-dimensional display devices, and c) the navigational information is not visualised directly at the operation site, forcing the surgeon to obtain the navigational information from a location unrelated to his surgical workspace.

Augmented reality (AR) using stereoscopic display devices, e.g. head-mounted displays (HMDs), has been discussed in the community as an alternative three-dimensional visualisation technique for navigated surgery in the last decade by different groups $[1,2,3]$. In this approach the navigational information, derived from the same volumetric dataset, that is used in MPR based navigation, is superimposed onto the surgeon's view of the real world.

Navigation systems based on MPR or AR, respectively, have been presented as concurrent approaches and were never compared in evaluations using the same experimental setup. We implemented a MPR based navigation interface displayed on a computer monitor and an AR navigation interface displayed on a HMD. Furthermore, we propose to fuse both interfaces into a single threedimensional user interface and developed a hybrid navigation interface as a combination of both technologies. In order to evaluate the possible advantages of a hybrid navigation interface compared to its two complementary components, we conducted an experiment on the performance of computer guided instrument placement with three surgeons of different levels of experience.

\section{Materials and methods}

We use an optical tracking system with four cameras fixed to the ceiling for precise real-time localisation of the instrument and the surgical object. The tracking system is capable of tracking the targets in our setup with an accuracy of $<0.35[\mathrm{~mm}]$ RMS. The MPR-navigation system uses an off-the-shelf PC for visualisation (Fig. 2(b)). The AR system uses a stereoscopic video see-through HMD similar to a system described by Sauer et al. [3]. The display is equipped with two colour cameras to obtain images of the observed scene and a tracking camera, rigidly attached to the colour cameras, for head pose estimation using a marker frame as a reference (Fig. 2(a) $(\mathrm{G})$ ). The reason for the preference of a video-see-through display to an optical-see-through device is, first of all, that these systems achieve a perfect synchronisation of video and head pose data since all cameras are genlocked, eliminating any lag between the images of the cameras. Secondly, we have more options for merging virtual and real objects, while optical systems offer only a brightening augmentation.

The transformation from the coordinate system of the external tracking device to the two-dimensional coordinates in the overlay image is given by

$$
H_{\text {Target }}^{\text {Overlay }}=H_{\text {Cam }}^{\text {Overlay }} H_{\text {Frame }}^{\text {Cam }}\left(H_{\text {Frame }}^{\text {Ext }}\right)^{-1} H_{\text {Target }}^{\text {Ext }}
$$

The transformations $H_{\text {Frame }}^{\text {Ext }}, H_{\text {Target }}^{\text {Ext }}$ are provided by the external tracking system. $H_{\text {Frame }}^{\text {Cam }}, H_{\text {Cam }}^{\text {Overlay }}$ are derived using Tsai calibration. The instrument was calibrated and the patient registered as described in [4]. 
Fig. 1. Illustration of the experimental setup. X-ray dense, IR reflecting markers (E) attached to the phantom (D) and the drill (C) are tracked with an external optical tracking system (A). The visualisation (B) is displayed on an monitor, shown in figure (b) and respectively on the HMD (F) in figure (c). In the AR-system (a) a reference frame $(\mathrm{G})$ is used to establish the transition between the external tracking system (A) and the single camera tracking system of the HMD (F)

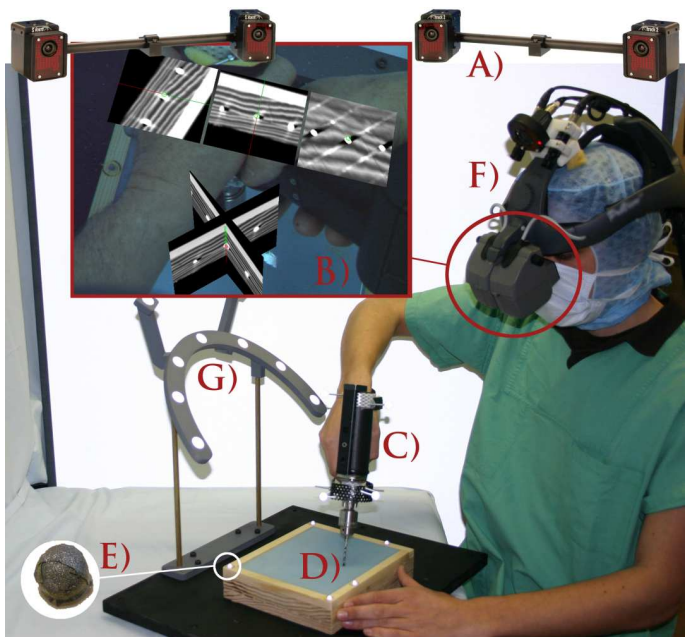

(a) Setup of the in-situ visualisation

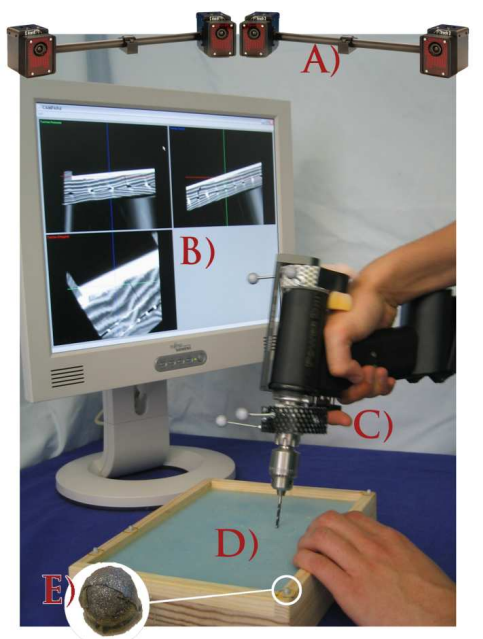

(b) Setup of the MPR-navigation

To achieve a fully automatic patient registration we used a reference target consisting of fiducials, that are automatically detectable in the imaging data and using the external tracking system, in the physical space.

The navigation interfaces were implemented using a software framework [5] that ensures sychronisation of video and tracking data, eliminating any perceivable drift of the augmentation. The three different navigation methods designed for this study are shown in figure 2 . We have decided to employ a in-situ visualisation method where two orthogonal slices are rendered along the longitudinal axis of an instrument, as this is, according to previous studies [6], a convenient guidance aid, especially regarding lateral positioning.

\section{Experiment and result}

A phantom that consists of a wooden block with metal spheres of $4[\mathrm{~mm}]$ diameter implanted at depth of approximately $40[\mathrm{~mm}]$ was constructed. The surface was covered with a silicone rubber compound with properties similar to human skin. We estimated a maximum target registration error (TRE) of the implanted metal spheres of $0.24[\mathrm{~mm}]$ and a mean error of $0.17[\mathrm{~mm}] \pm 0.03[\mathrm{~mm}]$.

Three surgeons conducted the experiment ten times for each of the three navigation methods. The distance between the surface of the implanted metal sphere and the distal end of the drill was recorded along with the time required to 
Table 1. Results of an experiment conducted by surgeons, regarding speed and accuracy. The navigation methods evaluated are monitor based navigation (MPR), in-situ visualisation (AR) and a hybrid combination of both (HYBRID). * denotes an experiment performed with the same surgeons under comparable conditions [6]

\begin{tabular}{lcccccc}
\hline Navigation & $\begin{array}{c}\text { Surgeon A } \\
\text { error }[\mathrm{mm}]\end{array}$ & time $[\mathrm{s}]$ & $\begin{array}{c}\text { Surgeon B } \\
\text { error }[\mathrm{mm}]\end{array}$ & time $[\mathrm{s}]$ & $\begin{array}{c}\text { Surgeon C } \\
\text { error }[\mathrm{mm}]\end{array}$ & time $[\mathrm{s}]$ \\
\hline MPR & $0.75 \pm 0.68$ & $71 \pm 35$ & $1.12 \pm 0.52$ & $66 \pm 36$ & $0.58 \pm 0.44$ & $51 \pm 11$ \\
$\mathrm{AR}$ & $0.84 \pm 0.48$ & $47 \pm 17$ & $0.69 \pm 0.44$ & $48 \pm 21$ & $0.66 \pm 0.49$ & $29 \pm 8$ \\
HYBRID & $0.58 \pm 0.44$ & $48 \pm 14$ & $0.97 \pm 0.46$ & $63 \pm 20$ & $0.68 \pm 0.44$ & $30 \pm 9$ \\
\hline AR $^{*}$ & $2.3 \pm 0.9$ & $98 \pm 24$ & $1.7 \pm 0.6$ & $57 \pm 52$ & $3.1 \pm 2.9$ & $47 \pm 28$ \\
HYBRID $^{*}$ & $1.6 \pm 0.6$ & $95 \pm 28$ & $1.9 \pm 0.5$ & $84 \pm 17$ & $1.9 \pm 0.2$ & $26 \pm 12$ \\
\hline
\end{tabular}

position the drill and reach the given target region. The results of the experiment are summarised in table 1.

Subject $\mathrm{A}$ is an inexperienced surgeon, who is not familiar with navigated surgery, subject B is a chief surgeon for trauma surgery, who performs navigated surgeries on a regular basis and subject $\mathrm{C}$ is a surgeon, who performs navigated surgeries and is involved in the development of systems for navigated surgery, that utilise AR technologies.

All experiments were performed within the accuracy of $1[\mathrm{~mm}]$ using a rigid, non deformable phantom. This result is acceptable according to the accuracy requirements for applications in orthopedic and trauma surgery. In order to be able to apply our methods to computer aided surgery however, additional challenges have to be accomplished to achieve the same accuracy for tracking and registration as we do in our lab environment.

\section{Conclusion}

The experiment indicates that a monitor based navigation interface has several disadvantages compared to in-situ visualisation. It is not intuitively usable, especially for surgeons, who never performed a computer assisted surgery, since it

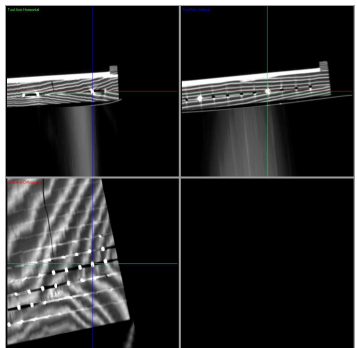

(c) MPR navigation

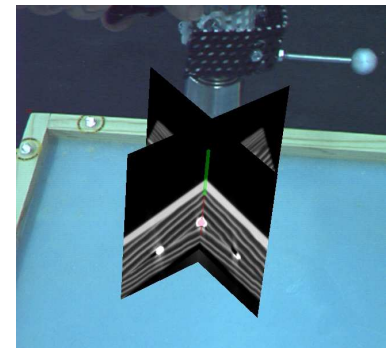

(d) In-situ visualisation

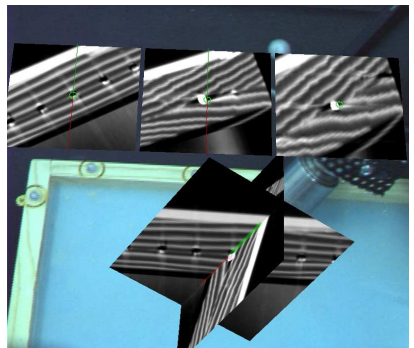

(e) Hybrid visualisation

Fig. 2. Different navigation modes displayed on a monitor (c) and in a HMD (d), (e) 
requires enormous comprehensive skills to mentally transfer the obtained information to the surgical environment. The experiment supports our thesis, that AR visualisation does not improve accuracy of surgical interventions, but, due to a more intuitive interaction, their speed. Throughout the experiment all three surgeons preferred the hybrid interface, that was described by them as intuitive and as accurate and safe as the monitor based system. The results affect our future research in the field of surgical navigation with AR technologies. We believe that a successful integration of surgical navigation in the operation theatre strongly depends on the intuitiveness of the user interface. Augmented reality can be a step towards fulfilling this premise. The presented hybrid navigation interface can benefit from the intuitiveness of in-situ visualisation and the well proven accuracy of conventional navigation.

\section{Acknowledgment}

Special thanks to Frank Sauer, Ali Khamene and Sebastian Vogt from Siemens Corporate Research for providing us with the RAMP system. Additionally, we would like to thank Josef Schweiger for his help on the design of the phantom. Last but not least, we would like to thank Stefan Wiesner and K. Schweiger for moral support and company. This work was granted by the BFS within the NARVIS project (www.narvis.org).

\section{References}

1. King AP, Edwards PJ, Maurer CR Jr, de Cunha DA, Hawkes DJ, Hill DLG, et al. A system for microscope-assisted guided interventions. IEEE Trans Med Imag 2000;19(11):1082-1093.

2. Birkfellner W, Figl M, Huber K, Watzinger F, Wanschitz F, Hummel J, et al. A head-mounted operating binocular for augmented reality visualization in medicine: Design and initial evaluation. IEEE Trans Med Imag 2002;21(8):991-997.

3. Sauer F, Khamene A, Bascle B, Rubino GJ. A head-mounted display system for augmented reality image guidance: Towards clinical evaluation for iMRI-guided neurosurgery. In: Proc. of MICCAI. Springer-Verlag; 2001. 707-716.

4. Traub J, Stefan P, Heining SM, Sielhorst T, Riquarts C, Euler E, et al. Towards a hybrid navigation interface: Comparison of a slice-based navigation system with in-situ visualization. In: Proc. of MIAR. Springer-Verlag; 2006.

5. Sielhorst T, Feuerstein M, Traub J, Kutter O, Navab N. CAMPAR: A software framework guaranteeing quality for medical augmented reality. In: Proc. of CARS; 2006.

6. Traub J, Stefan P, Heining SM, Sielhorst T, Riquarts C, Euler E, et al. Hybrid navigation interface for orthopedic and trauma surgery. In: Proc. of MICCAI. SpringerVerlag; 2006. 373-380. 